\title{
Obstructive sleep apnea in diabetic patients
}

\author{
Prashanth K. Narukulla ${ }^{1}$, Narendra K. Narahari ${ }^{2, *}$, Bhaskar Kakarla ${ }^{3}$, Paramjyothi GK. ${ }^{4}$ \\ ${ }^{1}$ Senior Resident, ${ }^{\mathbf{2 , 3}}$ Associate Professor, ${ }^{4}$ Professor \& HOD, Dept. of Pulmonary Medicine, Nizam's Institute of Medical \\ Sciences, Hyderabad, Telangana, India
}

*Corresponding Author:

Email: drnarendra_nims_pulmonologist@yahoo.com

\begin{abstract}
Introduction: Diabetes mellitus (DM) and obstructive sleep apnea (OSA) are common disorders that often coexist. OSA may be an under recognized co-morbidity of DM. The purpose of this study is to estimate the proportion of DM patients who are at risk for OSA and describe factors associated with the risk

Material and Methods: An institutional based study from November 2013 to November 2014 included 50 cases of DMthat were significant for modified Berlin questionnaire. All the 50 cases were subjected to thorough clinical examination and routine investigations like complete blood counts (CBC), blood sugar levels, polysomnography, lipid profile, thyroid profile and modified Berlin questionnaire.

Results: A total of 50 DM patients were included in the study, out of which $29(58 \%)$ were male and 21(42\%) were female patients. Mean age of the patients was $51.52 \pm 11.97$ years and mean BMI was $30.20 \pm 9.41 \mathrm{~kg} / \mathrm{m} 2$, mean waist circumference was $90.22 \pm 7.58 \mathrm{~cm}$ and $25(50 \%)$ of patients were obese. All those DM patients with significant Berlin questionnaire score underwent an overnight polysomnography to confirm the diagnosis of OSA. It was found that $35(70 \%)$ out of 50 patients screened had apnea-hypopnea index $(\mathrm{AHI}) \geq 5$.

Conclusion: More than half of adults with DM at high risk for OSA, and many may be undiagnosed. In a primary care setting, the modified Berlin Questionnaire is an easily applied screening tool that identifies patients at increased risk of OSA who may benefit from further diagnostic studies and treatment of OSA. All DM patients should be screened with modified Berlin questionnaire. Control of DM and Body mass index will reduce apnea hypopnea index.
\end{abstract}

Keywords: Obstructive Sleep Apnea; Berlin Questionnaire; Diabetes mellitus; Polysomnography.

\section{Introduction:}

Sleep apnea is a sleep disorder characterized by pauses in breathing or periods of shallow breathing during sleep. There are three forms of sleep apnea: obstructive (OSA), central (CSA), and a combination of the two called mixed. OSA is the most common form. Risk factors for OSA include being overweight and obesity, a family history of the condition, allergies, a small airway, and enlarged tonsils [1]. In OSA, breathing is interrupted by a blockage of airflow, while in CSA breathing stops due to a lack of effort to breathe. Obstructive sleep apnea (OSA) is a treatable sleep disorder characterized byrepetitive upper airway collapse, leading to oxygen de-saturation and sleep fragmentation [1]. Diabetes mellitus (DM) and obstructive sleep apnea (OSA) are common disorders that often coexist. There may also be a more complex relationship between these conditions in which an underlying metabolic disorder predisposes for both or in which metabolic and autonomic abnormalities associated with one, influence the development of the other because both diabetes and OSA are associated with increased cardiovascular morbidity and mortality. It is possible that the presence of both conditions result in added or even synergistic health risks [1]. OSA has been identified as a highly prevalent co-morbidity of type 2 diabetes mellitus (DM), in particular, among obese patients with type 2 DM, which represent the vast majority ofindividuals with type 2 DM [2-4]. It is estimated that about $80 \%$ of cases are not diagnosed [5]. In the western population theprevalence of OSA in the middle-aged (30 to 60 years) is $4 \%$ in men and $2 \%$ in women [6]. However, very little literature is available about the prevalence of OSA in Indian population. A study done in Delhi estimated the prevalence of OSA and OSAHS in an Indian study population to be $13.7 \%$ and $3.6 \%$ respectively [7]. Although loud snoring is seen in all patients with OSA, not all snorers have OSA.Understanding the differences between patients with OSA and simple snorers is important to explain the mechanisms responsible for upper airway obstruction rather than those between OSA and normal non-snorers [8]. Polysomnography is considered to be the gold standard for diagnosis of OSA, estimation of its severity and measurement of treatment response. Screening of metabolic syndrome patients for undiagnosed OSA, such as day time symptoms like excessive day time somnolence and nocturnal snoring, neck circumference, micrognathia, retrognathia and high arched palate. Early diagnosis and treatment can prevent the potential complications of the disease.

\section{Aims and Objectives of the Study:}

1. To correlate duration of DM and severity of Obstructive Sleep Apnea.

2. Correlate relation between glycosylated hemoglobin and apnea-hypopnea index (AHI). 
3. Correlate relation between body mass index (BMI) and AHI.

4. Correlate severity of AHI and other co-morbidities associated with diabetes mellitus like hypertension, coronary heart disease, renal failure, cerebrovascular accidents.

5. To understand the correlation between diabetics with significant modified Berlin questionnairescore and diabetics with insignificant modified Berlin questionnaire score.

\section{Material and Methods:}

This was a hospital based cross-sectional study done in adult patients with DM who visited the Department of Pulmonary Medicine, Navodaya Medical College Hospital and Research Centre, Raichur, Karnataka, India over a period of 12 months (November 2013 to November 2014).

Inclusion criteria:

All diabetic patients above the age of 18 years.

Exclusion criteria:

1. All diabetic patients who are not consenting for sleep study.

2. All diabetic patients below age of 18 years.

3. Post-operative cases.

4. Patients with known space occupying lesions in abdomen, chest and cranium.

5. Alcoholics.

6. In individuals with severe gastrointestinal distress, frequent urination, neurological diseases such as restless leg syndrome.

7. In patients using insomnia causing drugs like alfuzosin, doxazosin, prazosin, silodosin, terazosin and tamulosin.

8. Pregnancy.

9. Critically ill patients.

Investigations required for the study:

1. Random Blood Sugar (RBS)

2. Fasting blood sugar (FBS)

3. Post- prandial blood sugar (PPBS)

4. Glycosylated hemoglobin (HbA1c)

5. Polysomnography (PSG) using Allengers polysomnography machine, Allengers Virgo SL32 of 32 leads which includes electroenchalography (EEG), electrocardiograpy (ECG), electrooculography (EOG), electromyography (EMG), abdominal movement, thoracic movement, limb movement, position, airflow, heart rate (HR), saturation $(\mathrm{SpO} 2)$.

6. Electrocardiography (ECG)

7. Lipid profile

8. Thyroid profile

9. Computed tomography (CT) or Magnetic Resonance Imaging (MRI) of headand neck in selected cases.

\section{Results:}

A total of $50 \mathrm{DM}$ patients were included in the study, out of which 29(58\%) were male and 21(42\%) were female patients. Most patients were in the age group 60-69 years (table 1) and mean age of the patients was $51.52 \pm 11.97$ years and the mean BMI was $30.20 \pm$ $9.41 \mathrm{~kg} / \mathrm{m}^{2}$, mean waistcircumference was $90.22 \pm 7.58$ $\mathrm{cm}$ and $25(50 \%)$ of patients were obese.

All those DM patients with significant Berlin questionnaire score underwent an overnight polysomnography to confirm the diagnosis. OSA clinical features varied from snoring to asymptomatic. Snoring (16 cases) was the most predominant clinical feature followed by witnessedapneas ( 8 cases) and excessive daytime sleepiness (19 cases); 4 cases were asymptomatic. The co-morbidities associated with diabetes in the patients of our study other than OSA were hypertension, coronary artery disease, cerebrovascular accidents, dyslipidemia and renal failure.

Table 1: Age distribution of diabetic patients with OSA

\begin{tabular}{|c|c|}
\hline \multicolumn{2}{|c|}{ Age Distribution in years } \\
\hline $18-29$ & 1 \\
\hline $30-39$ & 5 \\
\hline $40-49$ & 7 \\
\hline $50-59$ & 8 \\
\hline $60-69$ & 13 \\
\hline $70-79$ & 1 \\
\hline
\end{tabular}

In this study, it was found that $35(70 \%)$ out of 50 patients screened had $\mathrm{AHI} \geq 5$ (Table 2). OSA was mild $(\mathrm{AHI}>5-15)$ in 14 cases, moderate $(\mathrm{AHI}>15-30)$ in 9 and severe $(\mathrm{AHI}>30)$ in 12 cases. Among the OSA in diabetics, 20 were male patients and 15 were female patients.

Table 2: Correlation of duration of DM with OSA and non OSA

\begin{tabular}{|l|c|c|}
\hline & OSA(AHI>5) & $\begin{array}{c}\text { Non OSA } \\
(\mathbf{A H I}<\mathbf{5})\end{array}$ \\
\hline $\begin{array}{l}\text { Duration of } \\
\text { DM<5 years }\end{array}$ & 5 & 2 \\
\hline $\begin{array}{l}\text { Duration of DM } \\
5-10 \text { years }\end{array}$ & 13 & 7 \\
\hline $\begin{array}{l}\text { Duration of } \\
\text { DM }>10 \text { years }\end{array}$ & 17 & 6 \\
\hline
\end{tabular}

In this study, when correlation between glycemic control in OSA and Non-OSA was made, it was found that most patients who had poor glycemic control had AHI $>5$ and severe OSA (Table 3). OSA was severe in patients who had BMI > 30 (Chart 1$)$.

Table 3: Glycemic control in OSA and non OSA patients

\begin{tabular}{|l|c|c|}
\hline & OSA(AHI $>\mathbf{5})$ & Non OSA $(\mathbf{A H I}<\mathbf{5})$ \\
\hline Hb1Ac $<7$ & 2 & 3 \\
\hline Hb1Ac $7-10$ & 18 & 13 \\
\hline Hb1AC $>8$ & 14 & 0 \\
\hline
\end{tabular}




\section{Chart 1: OSA and non OSA variation with BMI}

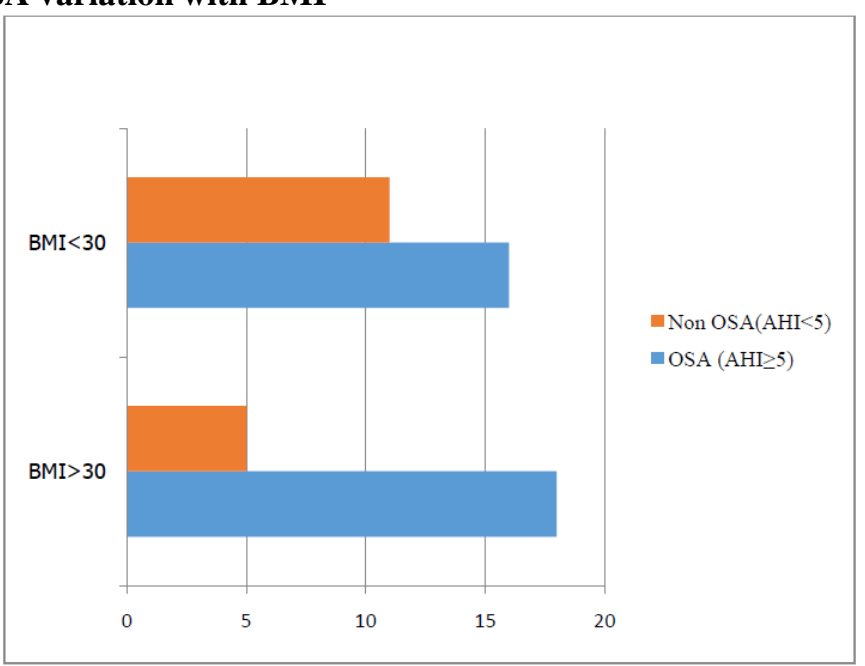

In this study, Berlin questionnaire risk score was done and it was found that in diabetics with poor glycemic control and AHI $>5$ had high risk when compared to AHI $<5$ and Non-OSA patients.

Table 4: Berlin questionnaire risk score in OSA and non OSA patients

\begin{tabular}{|l|c|c|}
\hline & OSA(AHI >5) & $\begin{array}{c}\text { Non OSA } \\
(\mathbf{A H I}<\mathbf{5})\end{array}$ \\
\hline $\begin{array}{l}\text { Berlin Questionnaire } \\
\text { score } \leq \text { 2(low risk) }\end{array}$ & 10 & 15 \\
\hline $\begin{array}{l}\text { Berlin Questionnaire } \\
\text { score>2(High risk) }\end{array}$ & 25 & 0 \\
\hline
\end{tabular}

\section{Chart 2:}

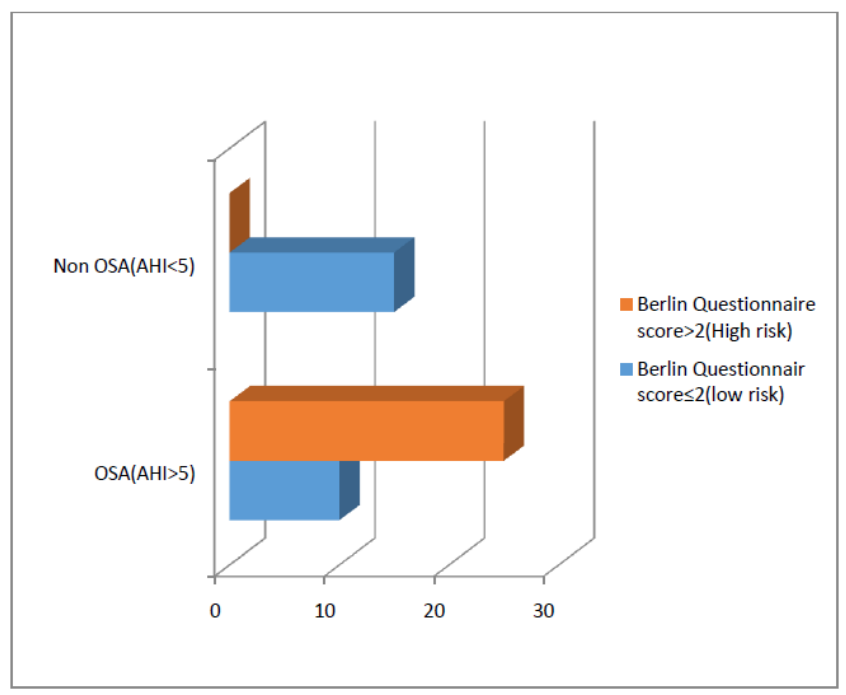

\section{Discussion:}

Our study indicates that OSA is highly prevalent in patients with DM. A total of 35 of our 50 DM subjects had OSA. These findings regarding the prevalence of OSA in DM are consistent with sleep AHEAD (Action for health in diabetes) study [9]. This study recruited 306 obese subjects with diabetes and found OSA prevalence of $86 \%$ using full overnight polysomnography, where BMI and waist circumference were identified as significant predictors of OSA. The study cohort was composed of obesesubjects (mean BMI, $36.5 \pm 5.8 \mathrm{~kg} / \mathrm{m}^{2}$ ), which could have contributed to highprevalence. Similar high prevalence of OSA up to $77 \%$ was reported in anotherclinical based study including 60 subjects with diabetes with mean BMI of $33.8 \mathrm{~kg} / \mathrm{m}^{2}$ [10]. The present study showed $70 \%$ prevalence of OSA in DM patients. Our study population included type-2 DMwith slight 
predominance of female sex. The majority were obese andhad a BMI $>30 \mathrm{~kg} / \mathrm{m}^{2}$. The high percentage of male patients with type-2 DM may explain the high prevalence of OSAS in males. OSAS has been considered predominantly disease of middle aged obese men and it is generally accepted that thispathology is twice as common in men as in women.Thesleep heart health study involving older DM individuals found OSA prevalence of $58 \%$, which is consistent with our study [6].

Our study has demonstrated a high prevalence of OSA in patients with DM. Many studies have proved the association of various co-morbidities in OSA with DM. It has also been proven that theprevalence of OSA is higher in patients with DM than in the general population or in obese non-DM subjects [11]. Our study is unique and different from other studies for being a hospital-basedprevalence study of OSA in patients with DM. The prevalence of OSA was found to be as high as $70 \%$, which is very high when compared with that reported in the general population in Indian as well as Western literature [12,13]. Our study, being a hospitalbased study wherein nearly one third of patients had already developed end organ damage as a result of DM and other co-morbidities could have had metabolic derangements for a longer duration. This could haveperpetuated a vicious cycle wherein the occurrence and severity of OSA among thesepatients could have been higher than that in the general population with OSA.

In our study, the age range of patients with DM and OSA was $27-71$ years. $74 \%$ patients were aged between 25-60 years and the remaining $26 \%$ were more than60 years of age. Two Studies by Bixler et al., [14] and the Sleep Heart Health Study [6] suggest that most of the age-related increase in prevalence of OSA occurs before age 65 and that there is a plateau in prevalence rate thereafter. Our study hasdemonstrated a similar trend in prevalence of OSA.In our study, 20 out of 29 (40\%)malesand 15 out of $21(30 \%)$ had OSA. Although there was a slight male preponderancein the OSA group, this was not statistically significant. In OSA prevalence studies, ithas been reported that OSA is 2 to 3 times more prevalent in men, particularly inmiddle aged obese men [12].

In our study, a similar gender ratio and predisposition to developing OSA wasobserved even when OSA and DM co-existed.In our study, all patients were obese and it was found that increasing grades ofobesity correlated well with presence of OSA. A higher waist circumference was noted in OSA when compared to the Non OSA group. Similarly, there was astatistically significant increased BMI in OSA group than in the Non OSA group. This observation was in concordance with many other studies which showed that obesity/BMI is one of strongest risk factors for OSA, which have been described below. In a community-based cohort of middle-aged subjects,
Young et al., [15] showed that a 1-SD increase in BMI was associated with a four-fold increased risk forprevalent sleep apnea, and they demonstrated a sleep apnea prevalence of approximately $40 \%$ in moderately overweight men from the community who were otherwise healthy. In severe obesity (BMI $>40 \mathrm{~kg} / \mathrm{m}$ 2 ), the prevalence of sleepapnea was estimated to vary between 40 and $90 \%$, and the severity of sleep apneawas generally greater than that found in leaner clinical populations. Peppard et al., [16] have provided further evidence for a link between sleep apnea and obesity bydemonstrating that a $10 \%$ change in body weight was associated with a parallelchange of approximately $30 \%$ in the apnea-hypopnea index (AHI), the major indexof sleep apnea severity. Markers of OSA severity, such as AHI index or the degree of oxygen de-saturation correlated with the amount of visceral fat in a study by Vgontzas et al., [17]. Measurement of neck circumference (NC) is a part ofthe physical examination of patients suspected of having sleep apnea. The mean neckcircumference in OSA patients in our study was higher than that of the non OSAgroup and the difference was statistically significant. Neck circumference of greaterthan 16 inches in a woman or greater than 17 inches in a man correlates with anincreased risk as it tends to make the retropharyngeal space shallow. Data from theWisconsin Sleep Cohort indicate that as little as a $10 \%$ increase in body weightincreases risk for OSA 6-fold [18]. One of the hypotheses proposed to explain thisincreased risk has been that excess adipose tissue around the upper airway increasesneck circumference and presents mechanical challenges for the lumen of the pharynxto stay patent during sleep [12].

Udwadia et al., [19] in his study among middleaged urbanIndian men reported that a significant proportion of their subjects were not obese byWestern or Asian standards but still had SDB. The explanation for this difference could be that pharyngeal narrowing, retrognathia or micrognathia, and pharyngealcollapsibility consequent to obesity might assume a greater pathogenic significance inIndian subjects. In our study, snoring was commonest symptom of OSA followedby EDS and witnessed apneas. There was a significant difference between the OSAand Non OSA groups in the incidence of these symptoms. Frequency of OSA among snorers was $45.71 \%$, that among patients with EDS was $54.29 \%$ and it was $22.86 \%$ in patients with witnessed apneas. Studies have showed that habitual snoring affects up to $50 \%$ of men and up to $30 \%$ of women in the general population [18]. Failure of medical providers to appreciate the impact of snoring on this population may affect attempts to identify, diagnose, and treat patients with sleep-disordered breathing. Snoring is also an important symptom for monitoring in long-term management of OSA, because its recurrence after OSA treatment may signal a need to re-evaluate the therapy. 
According to Young et al., (Wisconsin Sleep Cohort Study), habitual snoring occurs in $36 \%$ in adults and in more than $70 \%$ in subjects with an AHI of 5 or higher [18].

Snoring is probably the most common complaint precipitating a referral to a sleep clinic. Excessive daytime sleepiness and witnessed apneas were also found to be agood predictor of OSA by Lavie et al., [20]. In our study Berlin Questionnaire was used as a screening tool in DM patientspresenting with symptoms suggestive of OSA. The Berlin Questionnaire, an outcome of the Conference on Sleep in Primary Care held in Berlin, Germany in 1996 [21], was used to estimate the risk of obstructive sleep apnea, the primary outcomefor this study. Eleven items cover three domains related to risk of obstructive sleepapnea: (1) snoring and sleep-related symptoms, (2) awake-time sleepiness anddrowsiness, and (3) hypertension and/or a BMI greater than 30. Responses are codedpresent or absent for each category, and the final score ranges from 0 to 3 . Subjectswho score $\geq 2$ are classified as high risk. In our study, 25(71.4\%) patients had Berlin Questionnaire score $>2$ are10 (28.6\%) patients with Berlin Questionnaire score <2 with other symptomssuggestive of OSA, underwent overnight sleep study and were proven to haveOSA. The Berlin Questionnaire provided an estimate of the proportion of type 2 diabetics at high risk of obstructive sleep apnea (composite score $\geq 2$ ). In our study, all patients were DM and it was found that increasing grades ofobesity correlated well with presence of OSA. A higher waist circumference was noted in OSA when compared to the Non OSA group. Similarly, there was astatistically significant increased BMI in OSA group than in the Non OSA group.This observation was in concordance with many other studies which showed that obesity/BMI is one of strongest risk factors for OSA.

There are only a few published studies that have examined the association between the severity of OSA and the HbA1c levels. HbAlc is considered the major index for monitoring glycemic control in diabetic patients. Glycemic control in patients with DM is well known to be subjected to various DM-related factors, including anti diabetic medications, duration of DM, physical activity, and diet, which could interfere with any effect that OSA may pose. HbA1c does not reflect glucose fluctuations and might not be the major clinical indicator of glycemic control in the cases where frequent hypoglycemic and/or hyperglycemic episodes arepresent. Monnier et al., [22] demonstrated that glucose variability is a risk factor for complications independent of $\mathrm{HbAlc}$ in type $2 \mathrm{DM}$. To examine the impact ofuntreated OSA on glucose control in type 2 diabetes, Aron-sohn et al., [10] measured hemoglobin A1c in 60 consecutive patients with diabetes. OSA (AHI > 5) was present in 77\% of patients with type 2 diabetes and $38 \%$ of the patients had moderate or severe OSA (AHI > 15). Compared with patients without OSA, the adjusted meanhemoglobin A1C was increased by $1.49 \%$ in patients with mild OSA, $1.93 \%$ inmoderate OSA, and $3.69 \%$ in severe OSA.

OSA severity was associated with a poorerglucose control, independently of obesity and other confounding factors. Thesefindings were corroborated by a recent large multinational study, although in the analysis of the 6 participants of the European Sleep Apnea Cohort (ESADA) the adjusted mean HbA1c levels were only $0.72 \%$ higher in severe OSA patient's when compared with patients without OSA [9]. It was also found in this study that type 2 DM patients with a poor metabolic control had asignificant higher apneahypopnea index. On the other hand, it is possible that other confounding factors interferedwith $\mathrm{HbAlc}$ and did not let OSA to have an effect. West et al also did not find acorrelation between OSA severity and HbA1c in the subgroup of primary care patients [23].

A study of French men referred for assessment of sleep showed those with OSA (AHI > 10) were significantly more likely to have impaired glucose tolerance(IGT) and diabetes than were those without OSA [24]. The Sleep Heart Health Studyshowed a significant association between oxygen de-saturation during sleep and elevated fasting and 2-hour plasma glucose concentrations during an oral glucosetolerance test (OGTT). The severity of the OSA was also associated with the degreeof insulin resistance after adjustment for obesity. The Wisconsin Sleep Study $(n=1387)$ showed a significant cross-sectional association between OSA and type 2 diabetes for all degrees of OSA, which was preserved for those with moderate-severe OSA after adjustment for obesity (OR $=2.3$ ) [25]. The longitudinal data from the samestudy, however, showed that after adjusting for obesity, OSA at baseline was not asignificant predictor of the development of diabetes over 4 years. Thus, the present has demonstrated that there is a very high prevalence of OSA among the patients with DM compared to that in general population. The comorbidities associated with $\mathrm{DM}$, severity of DM, duration of DM are directly proportional to severity of OSA. Although a bigger study would have enable derivation of odds of developing OSA with DM or vice versa, there is convincing evidence of present study to mandatescreening for undiagnosed OSA in all DM patients.

\section{Conclusions:}

Our study involved low number of cases but still the results were satisfying; the incidence of OSA was significantly higher in Diabetes mellitus patients in comparison to general population. There was a significant association of co-morbidities between OSA and diabetes mellitus individually and in combination. Early detection and treatment of OSA in DM patients can prevent development of complications in them due to the combined effects of both diseases.Thus; the need 
for screening DM patients for undiagnosed OSA has been reinforced by this study.

Conflicts of interests: None declared Acknowledgements: None

\section{References:}

1. Fishman AP, Elias JA in Fishman's Pulmonary Diseases and Disorders. 2008;97:1571.

2. Einhorn D, Stewart DA, Erman MK, Gordon N, PhilisTsimikas A, Casal E. Prevalence of sleep apnea in a population of adults with type 2 diabetes mellitus. Endocrinol Pract 2007;13:355-62.

3. Resnick HE, Redline S, Shahar E, Gilpin A, Newman A, Walter R, et al. Diabetes and sleep disturbances: findings from the Sleep Heart Health Study. Diabetes Care 2003;26:702-9.

4. Foster GD, Sanders MH, Millman R, Zammit G, Borradaile KE,Newman AB, et al., Sleep AHEAD Research Group. Obstructive sleep apnea among obese patients with type 2 diabetes. Diabetes Care 2009;32:1017-19.

5. Punjabi NM, Shahar E, Redline S, Gottlieb DJ, Givelber $\mathrm{R}$, Resnick HE. Sleep-disordered breathing, glucose intolerance, and insulin resistance: the Sleep Heart Health Study. Am J Epidemiol 2004;160:521-30.

6. Young T, Palta M, Dempsey J, Skatrud J, Weber S, Badr $\mathrm{S}$. The occurrence of sleep disordered breathing in middle-aged adults. $N$ Engl J Med 1993;328(17):1230-5.

7. Sharma SK, Kumpawat S, Banga A, Goel A. The prevalence and risk factors of obstructive sleep apnea syndrome in a population of New Delhi, India. Chest 2006;130:149-56.

8. Yu X, Fujimoto K, Urushibata K, Matsuzawa Y, Kubo K. Cephalometric Analysis in Obese and Nonobese Patients With Obstructive Sleep Apnea Syndrome. Chest 2003;124:212-18.

9. Foster GD, Sanders MH, Millman R, Zammit G, Borradaile KE, Newman AB, et al, Sleep AHEAD Research Group. Obstructive sleep apnea among obese patients with type 2 diabetes. Diabetes Care 2009;32:1017-19.

10. Aronsohn RS, Whitmore H, Van Cauter E, Tasali E. Impact of untreated obstructive sleep apnea on glucose control in type 2 diabetes. Am J Respir Crit Care Med 2010;181:507-13.

11. Coughlin SR, Mawdsley L, Mugarza JA, et al. Obstructive sleep apnoea is independently associated with an increased prevalence of metabolic syndrome. Eur Heart J 2004;25:735.

12. Riha RL, Brander P, Vennelle M, McArdle N, Kerr SM, Anderson $\mathrm{NH}$, et al. The relationship between obesity and craniofacial structure in obstructive sleep apnea. Chest 1995; 108:375-81.

13. Smith PL, Gold AR, Meyers DA, Haponik EF, Bleecher ER. Weight loss in mildly to moderately obese patients with obstructive sleep apnea. Ann Intern Med 1985;103:850-5.

14. Bixler E, Vgontzas A, Ten Have T, Tyson K, Kales A. Effects of age on sleep apnea in men. Am J Respir Crit Care Med 1998;157:144-48.

15. Young T, Shahar E, Nieto FJ, Redline S, Newman AB, Gottlieb DJ, et al. Predictors of sleep-disordered breathing in community dwelling adults: the sleep heart health study. Arch Intern Med 2002;162:893-900.
16. Peppard PE, Young T, Palta M, Dempsey J, Skatrud J. Longitudinal study of weight change and sleep disordered breathing. JAMA 2000;284:3015-21.

17. Vgontzas AN, Bixler EO, Chrousos GP. Metabolic disturbances in obesity versus sleep apnoea: the importance of visceral obesity and insulin resistance. $J$ Intern Med 2003;254:32-44.

18. Young T, Finn L, Peppard PE, et al. Sleep disordered breathing and mortality: eighteen-year follow-up of the Wisconsin sleep cohort. Sleep. 2008;31(8):1071-8.

19. Udwadia ZF, Doshi AV, Lonkar SG, Singh CI. Prevalence of sleepdisordered breathing and sleep apnea in middle-aged urban Indian men. Am JRespir Crit Care Med 2004;169:168-73.

20. Peretz L. Incidence of sleep apnea in a presumably healthy working population: A significant relationship with excessive daytime sleepiness. Sleep: J Sleep Research \& Sleep Med 1983;6(4):312-18.

21. Netzer N, Stoohls R, Netzer C, Clark K, Strohl K. Using the Berlin Questionnaire to identify patients at risk for the sleep apnea syndrome. Ann Intern Med 1999;131(7):48591.

22. Monnier L, Colette C, Owens D. Glycemic variability: the third component of the dysglycemia in diabetes. Is it important? Howto measure it? J Diabetes Sci Technol 2008; 2:1094-100.

23. West SD, Nicoll DJ, Stradling JR. Prevalence of obstructive sleep apnoea in men with type 2 diabetes. Thorax 2006;61:945-50.

24. Meslier N, Gagnadoux F, Giraud P, et al., Impaired glucose-insulin metabolism in males with obstructive sleep apnoea syndrome, Eur Respir J 2003;22:156-60.

25. Reichmuth KJ, Austin D, Skatrud JB, et al., Association of sleep apnea and type II diabetes: a population-based study. Am J Respir Crit Care Med 2005;172:1590-95.

How to cite this article: Narukulla P. K., Narahari N. K., Kakarla B., GK. Paramjyothi. Obstructive sleep apnea in diabetic patients. IP Indian $J$ Immunol Respiratory Med 2018;3(3):131-136. 